\title{
Metalloelastase-12 is involved in the temporomandibular joint inflammatory response as well as cartilage degradation by aggrecanases in STR/Ort mice
}

\author{
YOKO YAMASHITA-FUTANI ${ }^{1}$, REI JOKAJI $^{1}$, KAZUHIRO OOI $^{1}$, \\ KAZUHIKO KOBAYASHI ${ }^{1}$, IOANNIS KANAKIS ${ }^{2}$, KE LIU $^{2}$, \\ SHUICHI KAWASHIRI ${ }^{1}$, GEORGE BOU-GHARIOS ${ }^{2}$ and HIROYUKI NAKAMURA ${ }^{1,3}$
}

\begin{abstract}
${ }^{1}$ Department of Oral and Maxillofacial Surgery, Kanazawa University Graduate School of Medical Science, Kanazawa, Ishikawa 920-8640, Japan; ${ }^{2}$ Institute of Ageing and Chronic Disease, University of Liverpool, Liverpool L7 8TX, UK;

${ }^{3}$ Department of Oral and Maxillofacial Surgery, Ryukyu University Graduate School of Medical Science, Nishihara, Okinawa 903-0215, Japan
\end{abstract}

Received December 26, 2020; Accepted March 17, 2021

DOI: $10.3892 /$ br.2021.1427

\begin{abstract}
Temporomandibular joint dysfunction (TMJD) is characterised by clinical symptoms involving both the masticatory muscles and the temporomandibular joint (TMJ). Disc internal derangement and osteoarthritis (OA) are the most common forms of TMJD. Currently, the molecular process associated with degenerative changes in the TMJ is unclear. Our previous study showed that elastin-digested peptides act on human TMJ synovial cells and lead to upregulation of interleukin-6 (IL-6) and metalloelastase-12 (MMP-12; an elastin-degrading enzyme) in vitro. However, there is limited information regarding the involvement of elastin-degradation by MMP-12 in the processes of inflammatory responses and cartilage degradation in vivo. STR/Ort mice were used as a model of TMJ OA in the present study. Significant articular cartilage degeneration was observed starting at 20 weeks of age in the STR/Ort mice and this progressed gradually until 40 weeks, compared with the age-matched CBA mice. Immunostaining analysis showed that MMP-12 and IL-6 were expressed in the chondrocytes in the superficial zones of the
\end{abstract}

Correspondence to: Professor Hiroyuki Nakamura, Department of Oral and Maxillofacial Surgery, Ryukyu University Graduate School of Medical Science, 207 Uehara, Nakagami, Nishihara, Okinawa 903-0215, Japan

E-mail: hnak@me.com

Abbreviations: TMJD, temporomandibular joint dysfunction; TMJ, temporomandibular joint; MMP-12, metalloelastase-12; OARSI, Osteoarthritis Research Society International, ADAMTS, a disintegrin and metalloproteinase with thrombospondin motifs; IL-6, interleukin-6; TNF- $\alpha$, tumour necrosis factor- $\alpha$; IL-1 $\beta$, interleukin-1 $\beta$

Key words: MMP-12, ADAMTS-4, ADATS-5, TMJ, STR/Ort, elastin, IL-6 cartilage. Immunostaining also showed that aggrecanases [a disintegrin and metalloproteinase with thrombospondin motifs (ADAMTS)-4 and ADAMTS-5] were expressed in the chondrocytes in the superficial zones of the cartilage. These findings suggest that an inflammatory and degradative process was initiated in the TMJ. Harmful mechanical stimuli, particularly pressure, may cause damage to the elastin fibres in the most elastin-rich superficial layer of the articular cartilage. Elastin-digested peptides are then generated as endogenous warning signals and they initiate a pro-inflammatory cascade. This leads to upregulation of pro-inflammatory mediators, such as IL-6 and MMP-12, which further trigger tissue damage resulting in elevated levels of elastin-digested peptides. IL-6 increases expression of the aggrecanases ADAMTS-4 and ADAMTS-5, following cartilage degradation. This leads to the establishment of a positive feedback loop and may result in chronic inflammation and cartilage degradation of the TMJ in vivo.

\section{Introduction}

The characteristics of temporomandibular joint dysfunction (TMJD) include musculoskeletal conditions and craniofacial pain in the masticatory system that involve the joint, masticatory muscles or muscle innervations. Various factors are associated with the aetiology of TMJD, including growth and developmental anomalies (1), trauma (2), detrimental body posture (3), parafunctional habits and bruxism (4), and stress (5). The most common forms of TMJD are disc internal derangement, which involves an abnormal anatomical relationship between the articular disc and articulating surfaces, and osteoarthritis (OA), which involves an abnormal anatomical relationship between the articular disc and articulating surfaces (6). Although the degenerative changes in the temporomandibular joint (TMJ) have been reported to be related to osteoclastogenesis (7), the molecular process underlying these changes are yet to be elucidated.

Previous studies (8-11) have reported increased concentrations of monocyte-macrophage-derived cytokines in the 
synovial fluid of patients with TMJD. The release of proteinases and stimulation of the expression of degrading enzymes and inflammatory mediators may be facilitated by various cytokines, including interleukin $1 \beta$ (IL-1 $\beta$ ), interleukin-6 (IL-6) and tumour necrosis factor- $\alpha$ (TNF- $\alpha$ ), thereby leading to inflammation of the TMJ and degradation of bone and cartilage (12). These results highlight the potential role of cytokines in TMJD pathogenesis. Our previous study showed that IL-6 expression in the synovial fluid obtained from patients with TMJD was significantly correlated with the two clinical parameters of TMJ locking and pain/jaw function based on a visual analogue scale (VAS) (13). Additionally, the concentration of elastin-digested peptides in the synovial fluid of patients with TMJD was significantly associated with the TMJ locking duration, the VAS and IL-6 expression (13). In vitro, elastin-digested peptides act on human TMJ synovial cells to promote IL-6 upregulation and MMP-12 (an elastin-degrading enzyme) (13). These findings suggest an inflammation model in the TMJ where elastin is degraded by a harmful mechanical stimulus, and the degradation products induce a pro-inflammatory cascade and increase the expression of MMP-12.

Currently, there is limited information regarding the involvement of elastin-degradation by MMP-12 in the process of inflammatory responses and cartilage degradation by aggrecanases [a disintegrin and metalloproteinase with thrombospondin motifs (ADAMTS)-4 and ADAMTS-5] in vivo. The STR/Ort mice often develop spontaneous OA of the medial tibial cartilage of the knee joint Thus this mouse model is useful in studying the pathogenesis of knee OA (14). The histopathological lesions of knee OA in STR/Ort mice are progressive and exhibit a high degree of similarity to that of human knee OA. Moreover, $85 \%$ of STR/Ort mice demonstrate histological OA lesions in the medial tibial cartilage by the time they are 35 weeks old. Previous reports have shown that the STR/Ort mice develop spontaneous OA-like lesions in the TMJ with age, and thus, they may facilitate the study of the pathogenesis of TMJ OA (15). The aim of the present study was to investigate the role of MMP-12 and aggrecanases in STR/Ort mice with TMJ OA.

\section{Materials and methods}

Assessment of the progression and severity of $O A$. A total of 24 male STR/Ort mice (age, 10 weeks) and 8 sex-matched CBA control mice were used in the present study. The average weight of the STR/Ort mice at the beginning of the experiment (age, 10 weeks) was $24.1 \pm 1.6 \mathrm{~g}$, whereas that of the CBA control mice (age, 10 weeks) was $25.3 \pm 1.4 \mathrm{~g}$. The mice TMJs were removed by microsurgery and were fixed in $4 \%$ paraformaldehyde solution for $24 \mathrm{~h}$ at room temperature. Decalcification of TMJs was performed using 10\% EDTA for 3 weeks at room temperature, and subsequently stored in $70 \%$ ethanol at $4^{\circ} \mathrm{C}$ until required for further analysis. Samples were embedded in paraffin wax and sectioned sagittally (5- $\mu \mathrm{m}$ thickness) through the entire joint. Safranin-O (Sigma-Aldrich; Merck $\mathrm{KGaA}$ ) staining was used at room temperature to evaluate proteoglycan loss using a validated scoring system as per the Osteoarthritis Research Society International (OARSI) recommendations (16). The joints were graded at $10-\mu \mathrm{m}$ intervals through the joint in a blinded manner by two experienced investigators, and were scored as follows: 0, normal cartilage; 0.5 , loss of Safranin-O without structural changes; 1, small fibrillations without loss of cartilage; 2, vertical clefts down to the layer immediately below the superficial layer and some loss of surface lamina; 3 , vertical clefts/erosion to the calcified cartilage extending to $<25 \%$ of the articular surface; 4 , vertical clefts/erosion to the calcified cartilage extending to $25-50 \%$ of the articular surface; 5 , vertical clefts/erosion to the calcified cartilage extending to $50-75 \%$ of the articular surface; and 6 , vertical clefts/erosion to the calcified cartilage extending to $>75 \%$ of the articular surface. Scores were added from all levels through the entire joint to obtain the 'summed score', which reflected the severity of the osteoarthritic lesion. OA predominantly affects the mandibular condyle (15); therefore, the average of the summed scores at both the sites was used for each sample to obtain robust results.

All the animal experiments performed in the present study were approved by the Ethics Committee of the Kanazawa University Graduate School of Medical Science (approval no. 352-2). The mice were housed in groups of four in individually vented cages maintained at $21^{\circ} \mathrm{C} \pm 2^{\circ} \mathrm{C}$ and a humidity range of $30-70 \%$ in a 12 -h light/dark cycle with ad libitum access to food and water. The animals were monitored daily for any health and/or welfare issues from the time of birth, including any possible defects or significant changes in size during the first 2 weeks of life. The mice were then sacrificed in a chamber by increasing the $\mathrm{CO}_{2}$ concentration at a flow rate of $60 \% / \mathrm{min}$ of volume. Lack of respiration and faded eye colour were used to confirm death.

Immunohistochemical analysis. The paraffin embedded sagittal sections of TMJs were dewaxed and antigen retrieval were performed using Immunosaver (FUJIFILM Wako Pure Chemical Corporation) for $45 \mathrm{~min}$ at $98^{\circ} \mathrm{C}$. The sections were washed in PBS, and then rinsed in PBS containing $1 \%$ BSA and $2 \%$ foetal calf serum. Immunohistochemical staining was performed with antibodies against MMP-12 (cat. no. 22989-1-AP; ProteinTech Group, Inc.), IL-6 (cat. no. ab6672; Abcam), ADAMTS-4 (cat. no. ab185722; Abcam) and ADAMTS-5 (cat. no. ab41037; Abcam). Primary antibodies were diluted in PBS at a ratio of 1:1,000, and the sections were then incubated with primary antibodies overnight at $4^{\circ} \mathrm{C}$. Binding of these antibodies was detected using EnVision Single Reagents (Dako; Agilent Technologies, Inc.). After washing, the slides were incubated with 3,3'-diaminobenzidine tetrahydrochloride (Sigma-Aldrich; Merck KGaA) and immediately rewashed under tap water following colour development. Slides were then counterstained with haematoxylin for $3 \mathrm{~min}$ at room temperature (FUJIFILM Wako Pure Chemical Corporation), mounted with DPX (FUJIFILM Wako Pure Chemical Corporation) and observed under a light microscope at a magnification of x4-x100 (Olympus Corporation). The specificity of staining was confirmed using a Universal Negative Control for IS-Series Rabbit Primary Antibodies (cat. no. IS60061; Dako; Agilent Technologies, Inc.) as a negative control, according to the manufacturer's protocol. Immunohistochemical staining was graded in a blinded manner by two experienced investigators as follows: 0 , no staining, 1; minor staining, 2; marked staining; and 3, maximal staining, as described previously (17). 
A

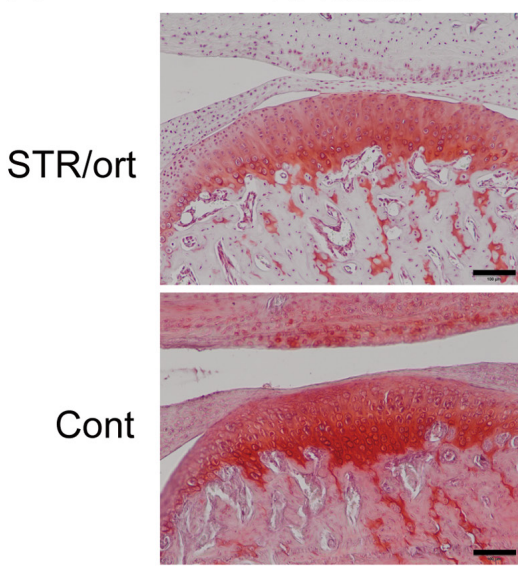

20 weeks

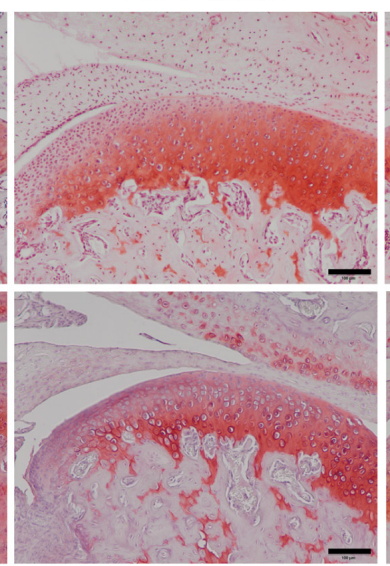

Safranin O

B

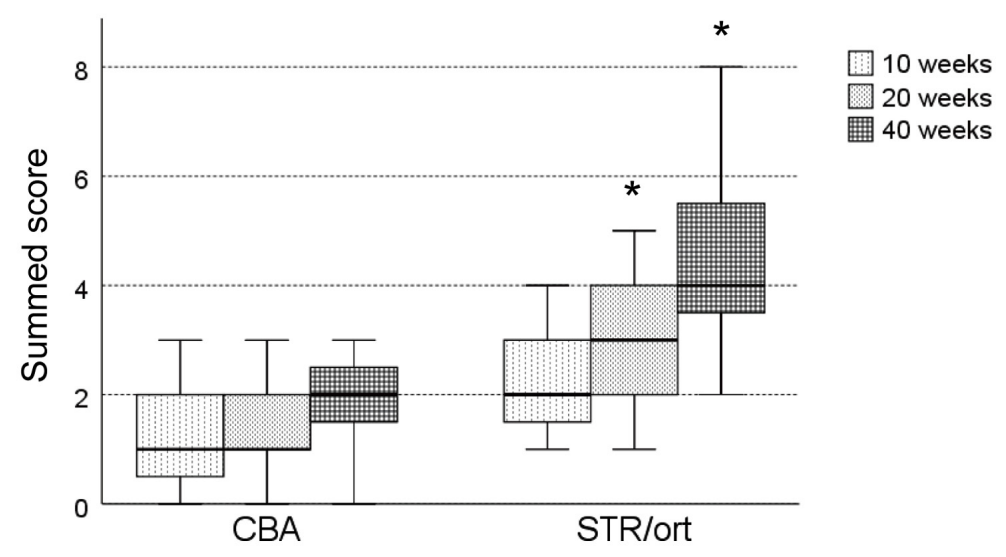

Figure 1. Cartilage degradation of the TMJ in the STR/Ort mice. (A) Safranin-O staining of sections of the TMJ of the STR/Ort mice and control CBA mice after 10, 20 and 40 weeks. Scale bar, $100 \mu \mathrm{m}$. (B) Box-whisker plots representing the summed OARSI scores of TMJs of the STR/Ort mice and control CBA mice after 10, 20 and 40 weeks. Results are expressed as the sum of the OARSI scores from each histological section through the joints. Values are expressed as the median \pm interquartile range. $n=8$ mice per group. Data were compared using Kruskal-Wallis one-way analysis of variance on ranks with a post-hoc Dunnett's test. ${ }^{*} \mathrm{P}<0.05$ vs. CBA control mice. TMJ, temporomandibular joint; OARSI, Osteoarthritis Research Society International; Cont, control.

Statistical analysis. The summed OARSI scores of joints are presented using a box-whisker plot (median \pm interquartile range). The immunohistochemical scores are presented as the mean \pm standard error of the mean. There were eight samples in both the STR/Ort and CBA groups. For comparisons between the samples, data were analysed using Kruskal-Wallis one-way analysis of variance on ranks with a post-hoc Dunnett's test for summed OARSI scores, or a Mann-Whitney U test for immunohistochemical scores using SPSS version 23 (IBM Corp.), as described previously $(17,18)$. $\mathrm{P}<0.05$ was considered to indicate a statistically significant difference.

\section{Results}

Cartilage degradation of the TMJs in the STR/Ort mice. In order to examine the pathogenesis of spontaneous OA, the TMJs of STR/Ort mice and control CBA mice were collected and sectioned after 10, 20 or 40 weeks. Relative to the age-matched control CBA mice, significant proteoglycan loss was observed in the upper zone of the cartilage after 20 weeks of age, and this loss was increased after 40 weeks (Fig. 1A). STR/Ort mice after 40 weeks exhibited vertical fissures in the matrix into the mid zone, and the fissures were branched (Fig. 1A). The data of the summed scores showed that significant articular cartilage degeneration started at 20 weeks of age in the STR/Ort mice and progressed gradually until they were 40 weeks old; this was not observed in the age-matched CBA mice (Fig. 1B).

Expression of MMP-12, IL- 6 and aggrecanases. Immunostaining showed that MMP-12 was expressed in the chondrocytes in the superficial zones after 20 weeks in the STR/Ort mice, and this increased gradually until the mice were 40 weeks old (Fig. 2A). In contrast, MMP-12 expression was detected in the chondrocytes in the CBA mice at all time points (Fig. 2A). In the subchondral bone, MMP-12 expression was observed in the osteoblast-like and osteoclast-like cells, starting at 20 weeks of age in the STR/Ort mice, and its levels increased with disease severity until the mice were 40 weeks old (Fig. 2A). However, no MMP-12 expression was detected in the subchondral bone of the CBA mice, regardless of age (Fig. 2A).

IL-6 was expressed throughout the cartilage of both the STR/Ort and CBA mice from 20 weeks of age, with expression in the chondrocytes in the superficial zones. However, the expression was significantly higher in the STR/Ort cartilage after 20 and 40 weeks relative to the age-matched CBA 
A
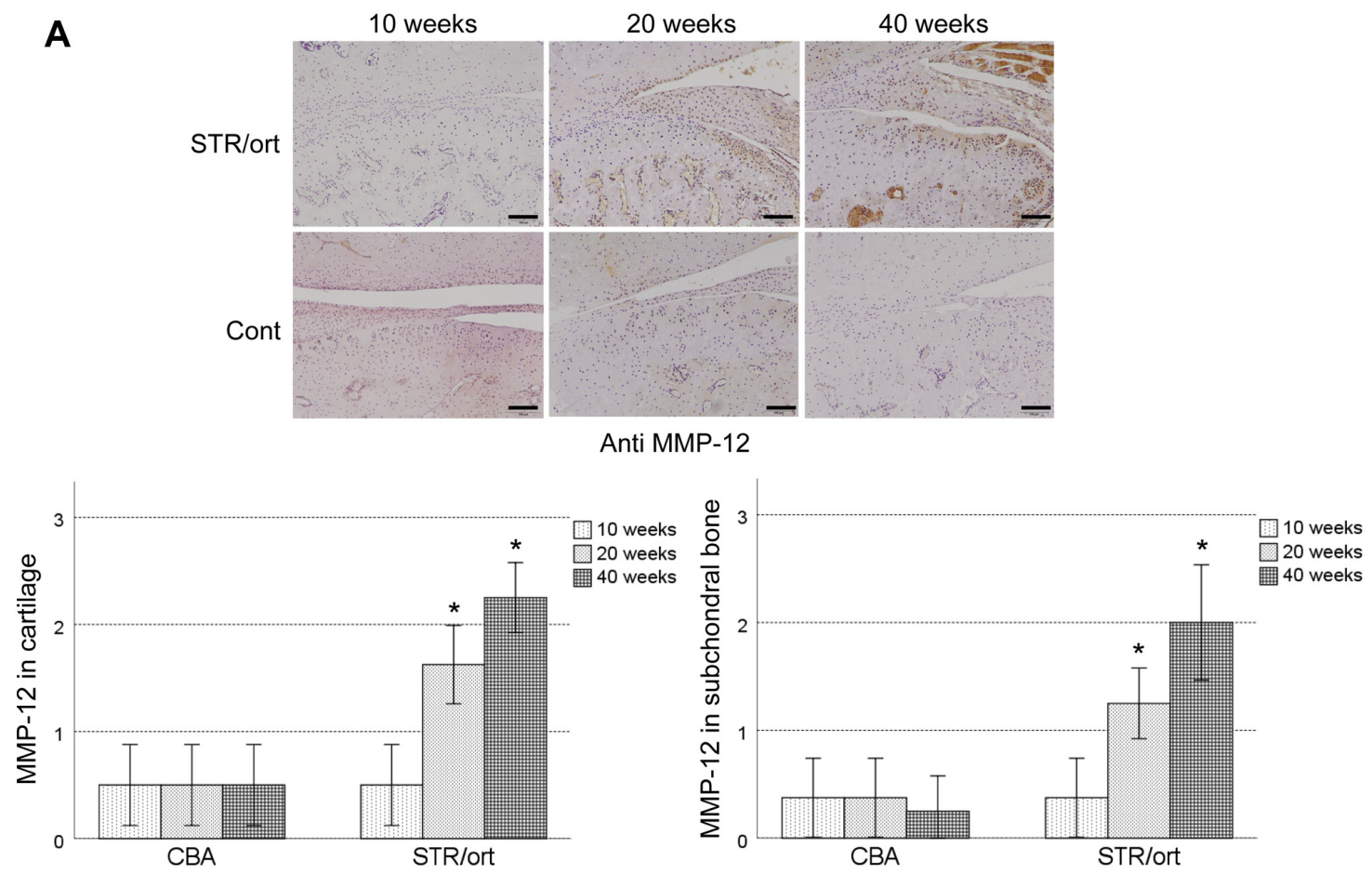

B
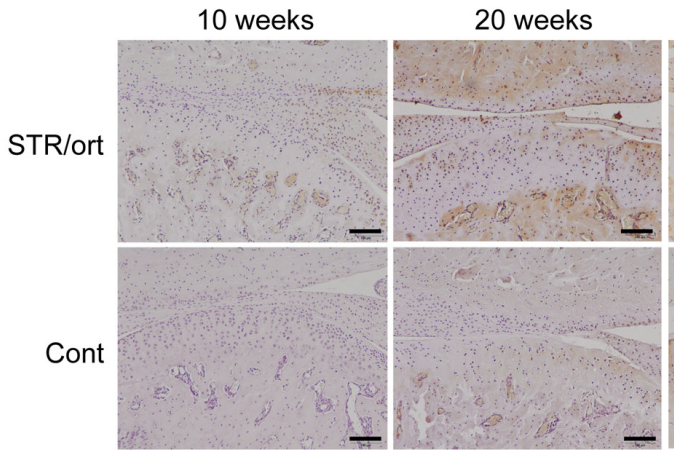

40 weeks

Anti IL-6
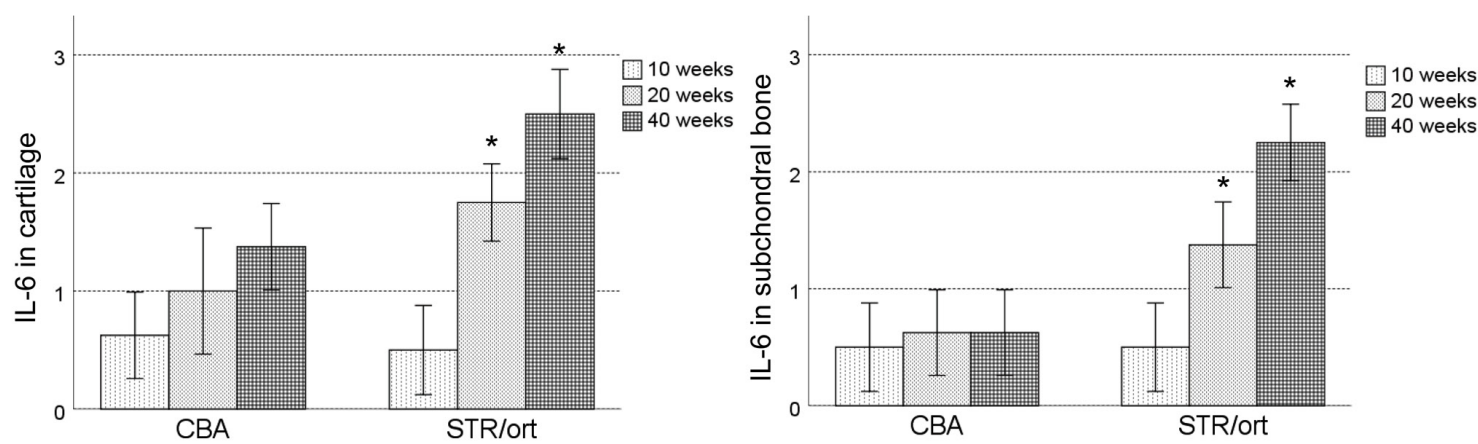

Figure 2. Expression of MMP-12 and IL-6 in the TMJ of STR/Ort mice. (A) MMP-12 or (B) IL-6 immunostaining of sections and the corresponding histological scores of the TMJs from STR/Ort mice and control CBA mice after 10, 20 and 40 weeks. The immunohistochemical scores in cartilage or subchondral bone are presented as the mean \pm the standard error of the mean. Scale bar, $100 \mu \mathrm{m}$. " $\mathrm{P}<0.05$ vs. CBA control mice. Mann-Whitney U test. MMP-12, metalloelastase-12; IL-6, interleukin-6; TMJ, temporomandibular joint.

mice (Fig. 2B). In the subchondral bone, IL-6 expression was observed in the osteoblast-like and the osteoclast-like cells, starting at 20 weeks of age in the STR/Ort mice, and its levels increased with disease severity until the mice were 40 weeks old (Fig. 2B). IL-6 expression in the subchondral bone in the STR/Ort cartilage was significantly higher compared with the age-matched CBA mice, excluding after 10 weeks (Fig. 2B). However, no detectable IL- $1 \beta$ or TNF- $\alpha$ expression was observed in the STR/Ort mice and CBA-strain mice, irrespective of age (data not shown).

ADAMTS-4 and ADAMTS-5 were expressed throughout the cartilage of both the STR/Ort and CBA mice from 20 weeks of age, with expression in the chondrocytes in the superficial zones that gradually increased till the mice were 40 weeks old (Fig. 3A and B). ADAMTS-5 was expressed in the middle zone of the cartilage after 40 weeks of age in 
A
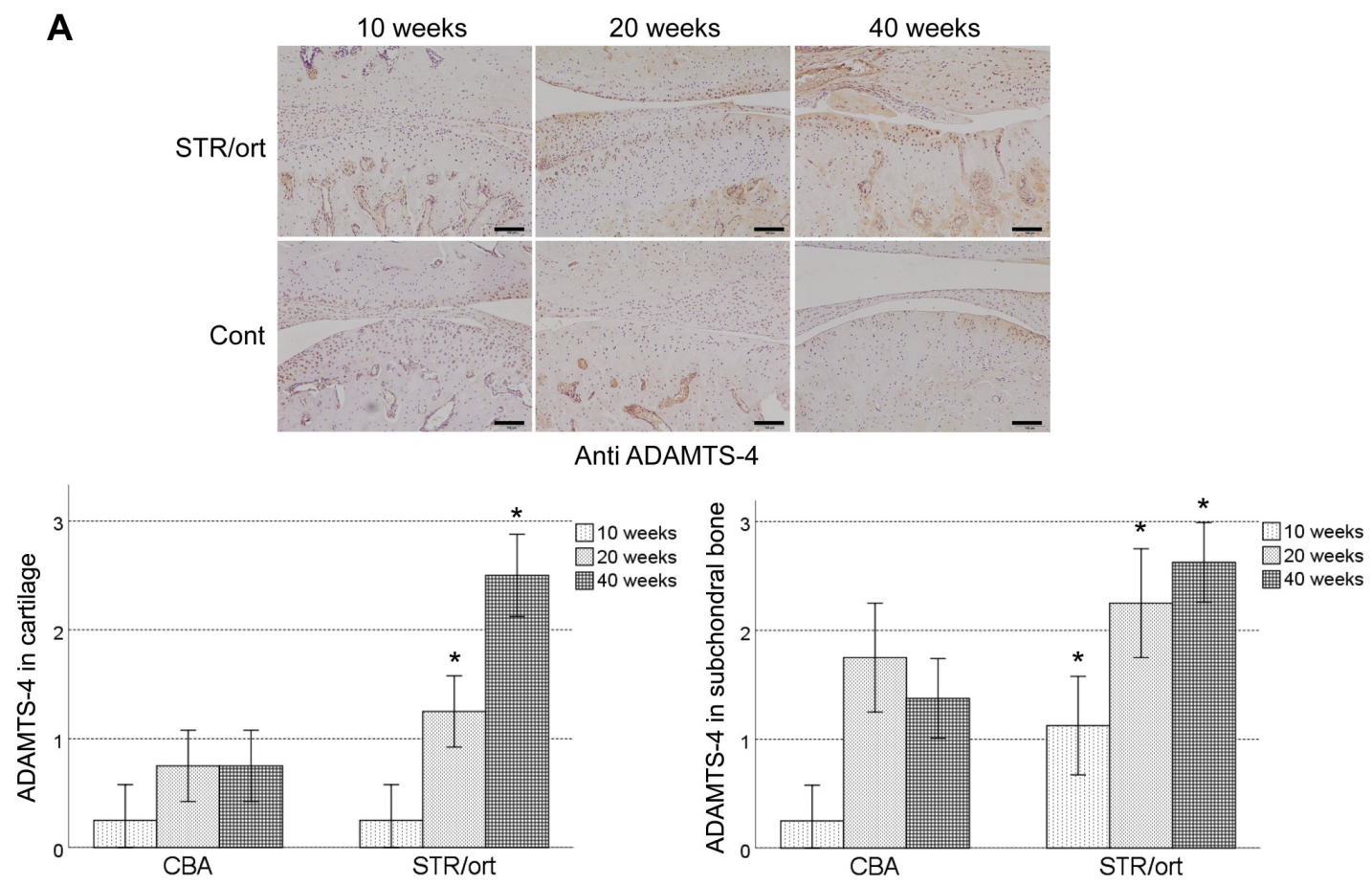

B

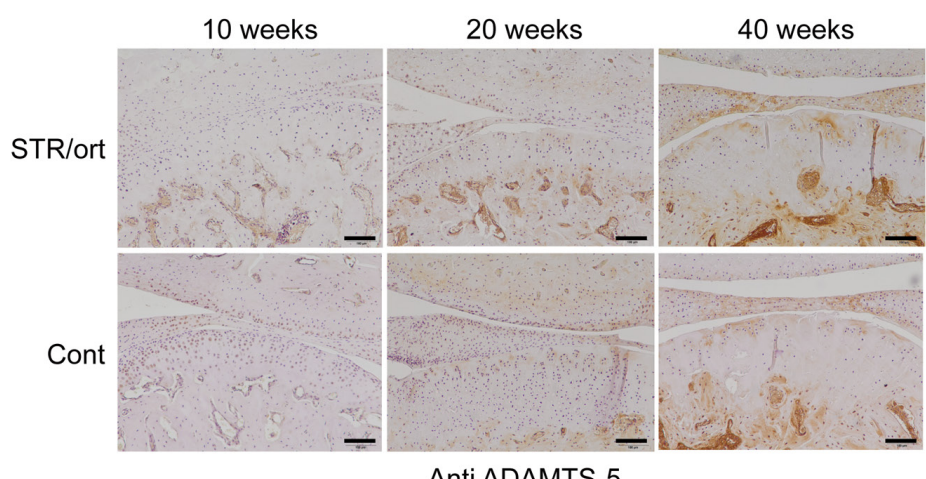

Anti ADAMTS-5
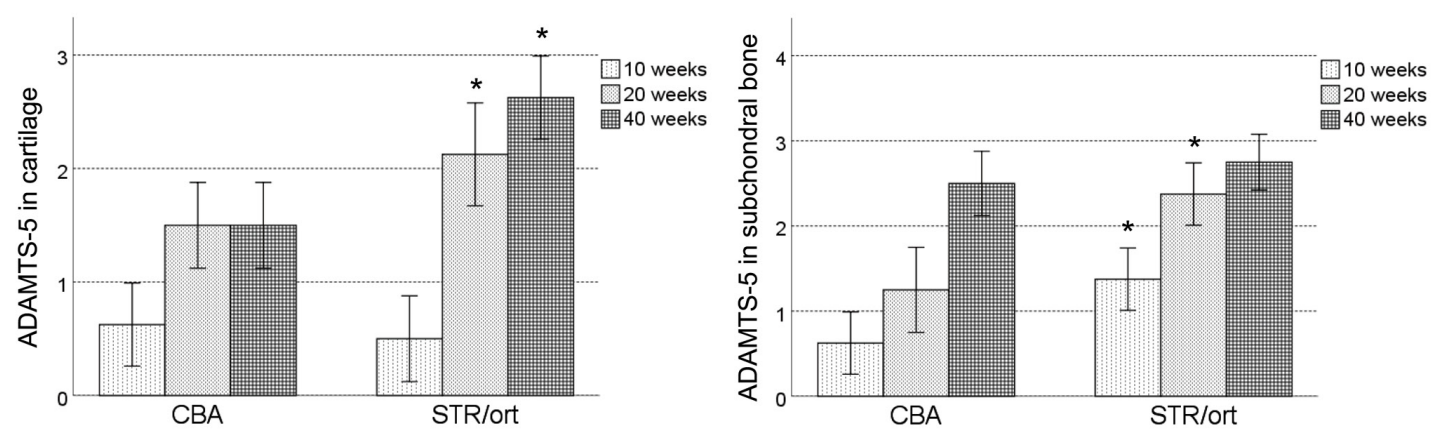

Figure 3. Expression of the aggrecan-degrading enzymes, ADAMTS-4 and ADAMTS-5, in the TMJ of STR/Ort mice. (A) ADAMTS-4 or (B) ADAMTS-5 immunostaining of sections and the corresponding histological scores of the TMJs of the STR/Ort mice and control CBA mice after 10,20 and 40 weeks. The immunohistochemical scores in the cartilage or subchondral bone are presented as the mean \pm standard error of the mean. Scale bar, $100 \mu \mathrm{m}$. Data were compared using a Mann-Whitney U test. ${ }^{*} \mathrm{P}<0.05$ vs. CBA control mice. ADAMTS, a disintegrin and metalloproteinase with thrombospondin motifs; TMJ, temporomandibular joint; Cont, control.

the STR/Ort mice. ADAMTS-4 and ADAMTS-5 expression was significantly higher in the STR/Ort cartilage compared with the age-matched CBA mice, excluding after 10 weeks (Fig. 3A and B). In the subchondral bone, ADAMTS- 4 and ADAMTS-5 expression was observed in the osteoblast-like and osteoclast-like cells, starting at 10 weeks of age in the STR/Ort mice, and its levels increased with disease severity until the mice were 40 weeks old (Fig. 3A and B).
ADAMTS-4 and ADAMTS-5 expression levels were significantly higher in the STR/Ort subchondral bone compared with the age-matched CBA mice (Fig. 3A and B).

\section{Discussion}

The primary aim of the present study was to determine whether elastin-degradation by MMP-12 was involved in 
TMJ inflammatory responses and cartilage degradation by aggrecanases in vivo. STR/Ort mice were used as a model of TMJ OA. The initial joint pathology caused by OA is difficult to characterise in humans as OA is often diagnosed in the first instance at an advanced stage. Animal models of OA may improve our understanding of the early pathology of OA, including loss of proteoglycans and fibrillations. Dreessen and Halata (19) showed age-related osteoarthritic degeneration of the TMJ in male STR/1N mice, suggesting a systemic basis of the disease. In the present study, significant articular cartilage degeneration began at 20 weeks of age in the STR/Ort mice and progressed gradually until 40 weeks relative to that in the age-matched CBA mice. The progression that was observed until 40 weeks of age in the STR/Ort mice was consistent with the results of a previous study (15). Conversely, in this same previous study, significant articular cartilage degeneration was not observed at 20 weeks of age in STR/Ort mice compared with the age-matched CBA mice (15). The maximal OARSI scores in STR/Ort mice at 20 and 40 weeks of age were 2 (highest OARSI score was 6). These results suggested that TMJ osteoarthritis at 20 and 40 weeks of age in STR/Ort mice showed initial or early joint pathology caused by OA. Thus, the STR/Ort mouse model may be appropriate for facilitating an understanding of the initial pathology of TMJ OA.

Elastin fibres are major extracellular matrix macromolecules that are critical for the maintenance of elasticity and resilience of tissues, such as blood vessels, lungs and skin. A previous study showed that the architecture of the elastin network varies significantly with cartilage depth (20). Dense elastin fibres formed a distinctive cobweb-like meshwork parallel to the cartilage surface in the most superficial layer of the articular cartilage (20). Contrastingly, in the superficial zone, the elastin fibres were found to be well organised in the physiological orientation, parallel to the collagen fibres. In the deep zone, no detectable elastin fibres were observed. In the elastin-containing superficial cartilage, inflammation was concomitant with elastolysis, leading to the generation of elastin-digested peptides (21-23). In these cases, a direct association between inflammation and elastin-digested peptide levels has been previously established (21-23). In the present study, immunostaining showed that MMP-12 was expressed in the chondrocytes in the superficial zones where elastin is found physiologically. These data suggest that MMP-12 directly digested elastin in the superficial zone of the cartilage and produced elastin-digested peptides. Our previous study demonstrated that the concentration of elastin-digested peptides in the synovial fluid of patients with TMJD was significantly correlated with IL-6 expression, the duration of TMJ locking and the VAS (13). IL-6 is generated at the inflammation site and plays a major role in the acute phase response (24). In the present study, immunostaining showed that IL-6 was expressed in the chondrocytes in the superficial zones where metalloelastase-12 is expressed during the initial and early joint pathology caused by OA. These data suggested that elastin peptides digested by MMP-12 induced IL-6 expression in the chondrocytes in the superficial zone of the cartilage. However, whether the elastin-digested peptides induced IL-6 expression directly in the chondrocytes, in vitro or in vivo, remains unclear. In this study, it was not possible to detect elastin-digested peptides in the joint fluid, as, if they were present, their levels were below the limits of detection. Further research using joint tissue-specific elastin-overexpressing mice or joint tissue-specific elastin knockout mice is warranted. In contrast, in the subchondral bone, MMP-12 expression was observed in osteoblast- and osteoclast-like cells in the 20-week-old STR/Ort mice and the frequency of occurrence increased with disease severity until the mice were 40 weeks old. In a previous study, it was shown that MMP-12 was present in the matrix adjacent to the osteoblast-like cells, bone lining cells and osteoclasts in moderate and severe stages of OA (25). In vitro and in vivo animal studies revealed that MMP-12 cleaved bone matrix proteins critical for osteoclast matrix interactions, and this was induced only in response to critical situations (26). However, elastin fibres have not been detected in the subchondral bone. A total of $90 \%$ of the organic matrix of bone is made of type I collagen, which is not degradable by MMP-12 (27). From a quantitative point of view, non-collagenous bone proteins, such as vitronectin, osteonectin, osteopontin and bone sialoprotein, are minimally present in the bone (26). It is thus interesting that vitronectin and osteonectin are completely degraded by MMP-12, but that osteopontin and bone sialoprotein are cleaved in a very selective way (28). Therefore, the degradation fragments of these minor bone proteins may potentially induce IL-6 expression in osteoblasts and osteoclasts. Further research is necessary to address this issue.

Following the onset of the inflammatory response, IL-6 secreted in the TMJ may function as a chemoattractant to recruit cell types that are crucial in tissue degradation (13). The stimulation of cartilage explants with IL-6 potentiated proteoglycan (aggrecan) catabolism in the articular cartilage has been previously reported (29). This catabolism was found to be associated with aggrecanase activity. The aggrecanases are members of the ADAMTS family of extracellular metzincin proteinases. Amongst the various members of this family of proteins, ADAMTS-4 and ADAMTS-5 show the highest levels of aggrecanase activity (30). Mice deficient in ADAMTS-5 were protected against aggrecan loss and cartilage degradation in models of inflammatory arthritis (31) and OA (32). In this regard, IL-6 upregulation is critical for the progression of arthritic diseases. IL- 6 contributes to cartilage destruction in mouse models of inflammatory arthritis $(33,34)$; as per one report, aggrecan loss is prevented in IL-6-deficient mice (34). IL-6 increases the mRNA expression levels of ADAMTS-4 and ADAMTS-5 in bovine chondrocytes (35). Moreover, IL-6-null mice are protected against cartilage damage both in the CIA model (36) and the antigen-induced arthritis model (33). In the present study, immunostaining revealed that ADAMTS-4 and ADAMTS-5 were expressed in chondrocytes in the superficial zones where IL-6 was expressed during the initial and early joint pathology caused by OA. These data suggested that IL-6 induced ADAMTS-4 and ADAMTS-5 expression in the chondrocytes, following cartilage degradation in the superficial zone in vivo.

In conclusion, a model for the initiation of the inflammatory and degradative process in the TMJ is proposed. Harmful mechanical stimuli, particularly increased pressure, may cause damage to the elastin fibres in the most elastin-rich superficial layer of the articular cartilage. Elastin-digested peptides 
are then generated as endogenous danger signals and induce a pro-inflammatory cascade. This leads to upregulation of pro-inflammatory mediators, such as IL-6 and MMP-12, triggering further tissue damage that results in increased levels of elastin-digested peptides. IL- 6 induced ADAMTS- 4 and ADAMTS-5 expression in the chondrocytes, following cartilage degradation. Thus, a positive feedback loop is established and may result in chronic inflammation and cartilage degradation of the TMJ in vivo.

\section{Acknowledgements}

Not applicable.

\section{Funding}

This study was supported by Grants-in-Aid for Scientific Research from the Ministry of Education, Science, Sports and Culture, Japan (grant nos. 18K09764, 18K17166, $15 \mathrm{H} 05042$ and $17 \mathrm{~K} 11869)$.

\section{Availability of data and material}

The datasets used and/or analyzed during the present study are available from the corresponding author on reasonable request.

\section{Authors' contributions}

HN was responsible for the conception and design of the study. YYF, RJ KK, IK and KL performed the experiments. YYF and HN wrote the manuscript. KO, GBG and SK assisted in the design of the study. HN edited the manuscript. All authors have read and approved the final manuscript. GBG and HN confirm the authenticity of all the raw data.

\section{Ethics approval and consent to participate}

All the studies using laboratory animals were approved by the Institutional Animal Care and Use Committees of Kanazawa University (approval no. AP183935). The protocols employed in the present study adhered to all applicable institutional and governmental guidelines for the humane care and use of laboratory animals.

\section{Patient consent for publication}

Not applicable.

\section{Competing interests}

The authors declare that they have no competing interests.

\section{References}

1. Power A and Carter L: Osteochondroma of the mandibular condyle: an unusual case of dentofacial asymmetry. Dent Update 42: 369-370, 372, 2015.

2. Nagori SA, Jose A, Bhutia O and Roychoudhury A: Undiagnosed mandibular condylar fractures causing temporomandibular joint ankylosis: A problem in northern India. Natl Med J India 27: 251-255, 2014
3. Durham J, McDonald C, Hutchinson L and Newton JL: Painful temporomandibular disorders are common in patients with postural orthostatic tachycardia syndrome and impact significantly upon quality of life. J Oral Facial Pain Headache 29: 152-157, 2015.

4. Sierwald I, John MT, Schierz O, Hirsch C, Sagheri D, Jost-Brinkmann PG and Reissmann DR: Association of temporomandibular disorder pain with awake and sleep bruxism in adults. J Orofac Orthop 76: 305-317, 2015.

5. Tosato JP, Caria PH, Gomes CA, Berzin F, Politti F, Gonzalez TO and Biasotto-Gonzalez DA: Correlation of stress and muscle activity of patients with different degrees of temporomandibular disorder. J Phys Ther Sci 27: 1227-1231, 2015.

6. Fang PK, Ma XC, Ma DL and Fu KY: Determination of interleukin-1 receptor antagonist, interleukin-10, and transforming growth factor-beta1 in synovial fluid aspirates of patients with temporomandibular disorders. J Oral Maxillofac Surg 57: 922-928, discussion 928-929, 1999.

7. de Bont LG, Boering G, Liem RS, Eulderink F and Westesson PL: Osteoarthritis and internal derangement of the temporomandibular joint: A light microscopic study. J Oral Maxillofac Surg 44: 634-643, 1986.

8. Shafer DM, Assael L, White LB and Rossomando EF: Tumor necrosis factor-alpha as a biochemical marker of pain and outcome in temporomandibular joints with internal derangements. J Oral Maxillofac Surg 52: 786-791, discussion 791-792, 1994.

9. Sandler NA, Buckley MJ, Cillo JE and Braun TW: Correlation of inflammatory cytokines with arthroscopic findings in patients with temporomandibular joint internal derangements. J Oral Maxillofac Surg 56: 534-543, discussion 543-544, 1998.

10. Sakamaki H, Ogura N, Kujiraoka H, Akiba M, Abiko Y and Nagura H: Activities of plasminogen activator, plasmin and kallikrein in synovial fluid from patients with temporomandibular joint disorders. Int J Oral Maxillofac Surg 30: 323-328, 2001.

11. Takahashi T, Kondoh T, Fukuda M, Yamazaki Y, Toyosaki T and Suzuki R: Proinflammatory cytokines detectable in synovial fluids from patients with temporomandibular disorders. Oral Surg Oral Med Oral Pathol Oral Radiol Endod 85: 135-141, 1998.

12. de Bont LG and Stegenga B: Pathology of temporomandibular joint internal derangement and osteoarthrosis. Int J Oral Maxillofac Surg 22: 71-74, 1993.

13. Kobayashi K, Jokaji R, Miyazawa-Hira M, Takatsuka S, Tanaka A, Ooi K, Nakamura H and Kawashiri S: Elastin derived peptides are involved in the processes of human temporomandibular disorder by inducing inflammatory responses in synovial cells. Mol Med Rep 16: 3147-3154, 2017.

14. Chambers MG, Cox L, Chong L, Suri N, Cover P, Bayliss MT and Mason RM: Matrix metalloproteinases and aggrecanases cleave aggrecan in different zones of normal cartilage but colocalize in the development of osteoarthritic lesions in STR/ort mice. Arthritis Rheum 44: 1455-1465, 2001.

15. Kumagai K, Suzuki S, Kanri Y, Matsubara R, Fujii K, Wake M, Suzuki R and Hamada Y: Spontaneously developed osteoarthritis in the temporomandibular joint in STR/ort mice. Biomed Rep 3: 453-456, 2015

16. Glasson SS, Chambers MG, Van Den Berg WB and Little CB: The OARSI histopathology initiative - recommendations for histological assessments of osteoarthritis in the mouse. Osteoarthritis Cartilage 18 (Suppl 3): S17-S23, 2010.

17. Valverde-Franco G, Pelletier JP, Fahmi H, Hum D, Matsuo K, Lussier B, Kapoor M and Martel-Pelletier J: In vivo bone-specific EphB4 overexpression in mice protects both subchondral bone and cartilage during osteoarthritis. Arthritis Rheum 64: 3614-3625, 2012

18. Nakamura H, Vo P, Kanakis I, Liu K and Bou-Gharios G: Aggrecanase-selective tissue inhibitor of metalloproteinase-3 (TIMP3) protects articular cartilage in a surgical mouse model of osteoarthritis. Sci Rep 10: 9288, 2020.

19. Dreessen D and Halata Z: Age-related osteo-arthrotic degeneration of the temporomandibular joint in the mouse. Acta Anat (Basel) 139: 91-96, 1990.

20. He B, Wu JP, Chen HH, Kirk TB and Xu J: Elastin fibers display a versatile microfibril network in articular cartilage depending on the mechanical microenvironments. J Orthop Res 31: 1345-1353, 2013.

21. Duca L, Lambert E, Debret R, Rothhut B, Blanchevoye C, Delacoux F, Hornebeck W, Martiny L and Debelle L: Elastin peptides activate extracellular signal-regulated kinase $1 / 2$ via a Ras-independent mechanism requiring both p110gamma/Raf-1 and protein kinase A/B-Raf signaling in human skin fibroblasts. Mol Pharmacol 67: 1315-1324, 2005. 
22. Houghton AM, Quintero PA, Perkins DL, Kobayashi DK, Kelley DG, Marconcini LA, Mecham RP, Senior RM and Shapiro SD: Elastin fragments drive disease progression in a murine model of emphysema. J Clin Invest 116: 753-759, 2006

23. Robinet A, Fahem A, Cauchard JH, Huet E, Vincent L, Lorimier S, Antonicelli F, Soria C, Crepin M, Hornebeck W, et al: Elastin-derived peptides enhance angiogenesis by promoting endothelial cell migration and tubulogenesis through upregulation of MT1-MMP. J Cell Sci 118: 343-356, 2005.

24. Gabay C: Interleukin-6 and chronic inflammation. Arthritis Res Ther 8 (Suppl 2): S3, 2006.

25. Kaspiris A, Khaldi L, Chronopoulos E, Vasiliadis E, Grivas TB Kouvaras I, Dagkas S and Papadimitriou E: Macrophage-specific metalloelastase (MMP-12) immunoexpression in the osteochondral unit in osteoarthritis correlates with BMI and disease severity. Pathophysiology 22: 143-151, 2015.

26. Hou P, Troen T, Ovejero MC, Kirkegaard T, Andersen TL, Byrjalsen I, Ferreras M, Sato T, Shapiro SD, Foged NT, et al: Matrix metalloproteinase-12 (MMP-12) in osteoclasts: New lesson on the involvement of MMPs in bone resorption. Bone 34: 37-47, 2004.

27. Chandler S, Cossins J, Lury J and Wells G: Macrophage metalloelastase degrades matrix and myelin proteins and processes a tumour necrosis factor-alpha fusion protein. Biochem Biophys Res Commun 228: 421-429, 1996.

28. Fu JY, Lyga A, Shi H, Blue ML, Dixon B and Chen D: Cloning, expression, purification, and characterization of rat MMP-12. Protein Expr Purif 21: 268-274, 2001

29. Flannery CR, Little CB, Hughes CE, Curtis CL, Caterson B and Jones SA: IL-6 and its soluble receptor augment aggrecanase-mediated proteoglycan catabolism in articular cartilage. Matrix Biol 19: 549-553, 2000.
30. Tortorella MD and Malfait AM: Will the real aggrecanase(s) step up: Evaluating the criteria that define aggrecanase activity in osteoarthritis. Curr Pharm Biotechnol 9: 16-23, 2008.

31. Stanton H, Rogerson FM, East CJ, Golub SB, Lawlor KE, Meeker CT, Little CB, Last K, Farmer PJ, Campbell IK, et al: ADAMTS5 is the major aggrecanase in mouse cartilage in vivo and in vitro. Nature 434: 648-652, 2005.

32. Glasson SS, Askew R, Sheppard B, Carito B, Blanchet T, Ma HL, Flannery CR, Peluso D, Kanki K, Yang Z, et al: Deletion of active ADAMTS5 prevents cartilage degradation in a murine model of osteoarthritis. Nature 434: 644-648, 2005. Erratum in: Nature 446: 102, 2007.

33. Ohshima S, Saeki Y, Mima T, Sasai M, Nishioka K, Nomura S, Kopf M, Katada Y, Tanaka T, Suemura M, et al: Interleukin 6 plays a key role in the development of antigen-induced arthritis. Proc Natl Acad Sci USA 95: 8222-8226, 1998.

34. Fosang AJ, Last K, Stanton H, Golub SB, Little CB, Brown L and Jackson DC: Neoepitope antibodies against MMP-cleaved and aggrecanase-cleaved aggrecan. Methods Mol Biol 622: 312-347, 2010.

35. Legendre F, Bogdanowicz P, Boumediene K and Pujol JP: Role of interleukin 6 (IL-6)/IL-6R-induced signal tranducers and activators of transcription and mitogen-activated protein kinase/extracellular. J Rheumatol 32: 1307-1316, 2005.

36. Sasai M, Saeki Y, Ohshima S, Nishioka K, Mima T, Tanaka T, Katada Y, Yoshizaki K, Suemura M and Kishimoto T: Delayed onset and reduced severity of collagen-induced arthritis in interleukin-6-deficient mice. Arthritis Rheum 42: 1635-1643, 1999.

This work is licensed under a Creative Commons

Attribution-NonCommercial-NoDerivatives 4.0 International (CC BY-NC-ND 4.0) License. 